\title{
Labyrinthe
}

19 | 2004 (3)

Le Bel Aujourd'hui

\section{Kenneth Burke, philosophie pratique}

\section{Franck Lemonde}

Traducteur : Franck Lemonde

\section{OpenEdition}

\section{Journals}

Édition électronique

URL : http://journals.openedition.org/labyrinthe/243

DOI : $10.4000 /$ labyrinthe. 243

ISSN : 1950-6031

Éditeur

Hermann

Édition imprimée

Date de publication : 15 décembre 2004

Pagination : 95-107

\section{Référence électronique}

Franck Lemonde, «Kenneth Burke, philosophie pratique », Labyrinthe [En ligne], 19 | 2004 (3), mis en

ligne le 19 juin 2008, consulté le 22 avril 2019. URL : http://journals.openedition.org/labyrinthe/243 ; DOI : 10.4000/labyrinthe.243

Propriété intellectuelle 


\title{
KENNETH BURKE, PHILOSOPHIE PRATIQUE
}

\author{
Traduction de Franck LEMONDE \\ franck_lemonde@hotmail.com
}

\section{Brève présentation}

L'œuvre de Kenneth Burke (1897-1993) est transversale à toutes les classifications courantes: traducteur (le public anglophone lui doit sa première version de La Mort à Venise de Thomas Mann, publiée à Boston par Bedford Books en 1924 sous le titre Death in Venice ${ }^{1}$ ), poète (Book of Moments, Poems 1915-1967, Berkeley, University of California Press, 1968), conteur (The White Oxen and Other Stories, UCP, 1968), c'est surtout comme critique littéraire (The Philosophy of Literary Form, UCP, 1941) et comme philosophe (la grande trilogie $A$ Grammar of Motives, A Rhetoric of Motives, Language as Symbolic Action, parus respectivement en 1945, 1950 et 1966, tous trois en UCP) qu'il se fit connaître outre-Atlantique. Lui-même aimait désigner sa propre tentative comme un «dramatisme, c'est-à-dire essentiellement une pensée de l'action, de ses conditions et de ses modalités, et plus particulièrement une pensée de cette «action symbolique» qui traverse le monde humain de part en part, à tel point que l'on peut dire qu'elle le constitue: le langage. "Nous sommes des corps qui apprennent le langage» est l'un de ses axiomes.

Il s'agira donc d'examiner l'inséparabilité (Burke est antidualiste) du physique et du linguistique, les effets physiques du langage ou les conséquences dans le langage des mouvements des corps. Il s'agira aussi de penser ce que c'est qu'apprendre, enseigner, endoctriner même, et surtout réapprendre, ne serait-ce que pour lutter contre les diverses formes d'endoctrinement que nous subissons et entretenons. En quoi le dramatisme est une politique: Burke a écrit en 1939 une

\footnotetext{
1. Au sujet de la traduction de Der Tod in Venedig de Thomas Mann, signalons que W. H. Auden, bon germaniste au demeurant et lui-même traducteur de Brecht, jugea l'essai de Burke « définitif ». Il a été republié en 1998, toujours par Bedford Books. (Toutes les notes sont du traducteur.)
} 
«Rhétorique du Mein Kampf de Hitler» dont il est difficile, sans vouloir, comme il le disait lui-même, prophétiser après l'événement, de ne pas admirer rétrospectivement la mise en garde, quoique inaudible en son temps. Cette rareté mise à part, Burke n'a cessé d'exercer son scepticisme sur les mécanismes rhétoriques du capitalisme et leurs implications tout à fait matérielles (voir le rôle de l'argent dans certains extraits traduits plus bas). Cette position ne le fit toutefois pas embrasser à pleine bouche le marxisme dogmatique de certains de ses proches, trop manichéen pour celui qui se présentait sans honte comme un augustinien (The Rhetoric of Religion: Studies in Logology, UCP, 1961).

Héritier prodigue d'une tradition critique peut-être inaugurée par la définition du scholar d'Emerson, dont il se réclame explicitement, à l'écart de la phénoménologie comme du positivisme logique et de leur neutralité scientiste vis-à-vis des Puissances, Burke rappelle encore à sa façon incongrue l'existence d'une pensée alternative, et même altérative, y compris aux États-Unis d'Amérique.

F. L.

\section{Le Langage comme action symbolique (1966)}

\section{Définition de l'homme ${ }^{2}$}

D'abord, quelques mots sur la définition en général. Je l'avoue: je vois dans une définition l'équivalent pour le critique du poème lyrique ou de l'aria dans l'opéra. En outre, on peut constater que, quand elle apparaît dans un essai, comme la définition de la tragédie d'Aristote dans sa Poétique, une définition récapitule la réalité de telle sorte que toutes les propriétés attribuées à la chose définie peuvent être comme «dérivées» de la définition. Dans le développement concret, la définition peut être ce sur quoi l'écrivain tombe en dernier. Ou bien elle peut être formulée en cours de route. Mais logiquement elle est antérieure aux observations qu'elle résume. Ainsi, comme tous les attributs de la chose définie répondent à la définition, la définition doit être considé-

2. Language as Symbolic Action, (UCP, 1966), Introduction, p. 3-18. 
rée comme «antérieure», dans cette acception purement non-temporelle de l'antériorité.

Les définitions sont aussi l'équivalent pour le critique du poème lyrique (même si un poète peut voir les choses autrement!) en ceci que l'écrivain, le plus souvent, «tombe dessus». Elles sont des «trouvailles » (breakthroughs), et sont par là même assez difficiles à dénicher. Nous devrions toujours recommencer à les éprouver, mais elles ne semblent pas toujours «bien marcher».

Une définition doit avoir juste assez de clauses, et pas davantage. Cependant, chaque clause doit être comme une tête de chapitre, sous laquelle des observations appropriées peuvent être assemblées, comme si elles en dérivaient.

Je propose ma définition de l'homme en espérant persuader le lecteur qu'elle fait bien l'affaire, ou sinon, l'inciter à décider ce qui devrait être ajouté, soustrait, ou modifié sur certains points.

\section{L'homme est l'animal qui se sert de symboles.}

Certes, ce n'est pas une grande surprise. Mais nous ne proposons pas notre définition en vertu d'une quelconque valeur paradoxale. Le but est d'obtenir un ensemble de clauses aussi exhaustif que possible et de méditer sur chacune d'elles. [...]

L'animal qui se sert de symboles, oui, évidemment. Mais pouvonsnous nous amener à réaliser simplement ce que cette formule implique, et combien l'écrasante majorité de ce que nous entendons par « réalité » a été élaborée pour nous par nul autre intermédiaire que nos systèmes de symboles? Enlevez nos livres : que reste-t-il de notre savoir sur l'histoire, la biographie, et même sur quelque chose d'aussi «terre à terre » que la position relative des mers et des continents? Qu'est-ce que notre «réalité » d'aujourd'hui (par-delà la ligne très ténue de nos vies individuelles) sinon tout ce fatras de symboles sur le passé, combiné avec tout ce que nous savons sur le présent, principalement par l'intermédiaire des cartes, des magazines, des journaux, etc.? À l'école, allant de classe en classe, les élèves passent d'un idiome à un autre. Les divers cours dans le cursus ne sont en réalité qu'autant de terminologies différentes. Et quelle que soit l'importance pour nous du petit bout de réalité dont nous avons tous eu une expérience de première main, «l'image» d'ensemble n'est qu'une construction de nos systèmes de symboles. 
Méditer sur ce fait, jusqu'à en voir toutes les conséquences, c'est un peu comme scruter, de l'autre côté du bord des choses (over the edge of things), le fond d'un abîme. Et c'est sans aucun doute l'une des raisons pour lesquelles l'homme, bien qu'étant typiquement l'animal qui se sert de symboles, adhère à une sorte de réalisme verbal naïf qui refuse de réaliser toute l'extension du rôle joué par la symbolicité ${ }^{3}$ dans ses conceptions de la réalité.

En répondant à des mots, et à leurs modes ouverts ou couverts de persuasion («progrès», qui fait souvent vibrer d'espoir, en est un exemple typique), nous tendons à oublier le genre de relation qui prévaut réellement entre le verbal et le non-verbal. Étant un lien entre nous et le non-verbal, les mots sont en même temps un écran qui nous sépare du non-verbal, quoique cette affirmation se retourne contre ellemême, puisqu'une bonne partie de ce «nous » séparé du non-verbal par le verbal n'existerait même pas sans le verbal (ou sans notre symbolicité en général, car il en va de même avec les systèmes de symboles de la danse, de la musique, de la peinture, etc.)

Une carte routière qui nous aide à nous orienter facilement d'un côté à l'autre du continent doit sa grande utilité à son exceptionnelle pauvreté ontologique. Elle nous dit extraordinairement peu de choses sur le voyage dont on va faire l'expérience avec force détails. En vérité, sa valeur pour nous réside très exactement dans son inanité essentielle. [...]

La deuxième clause est: inventeur du négatif. Je ne suis pas entièrement satisfait du mot «inventeur». Car nous ne saurions exactement dire que l'homme a «inventé» le négatif sans dire également que l'homme est l' «inventeur» du langage lui-même. En ce qui concerne son développement purement empirique, il serait plus précis de dire que le négatif a «inventé » l'homme. En tout cas, nous tenons compte ici du fait qu'il n'y a pas de négatifs dans la nature, et que cet ingénieux ajout à l'univers est uniquement un produit des systèmes symboliques humains. À une époque on l'on nous dit, même en chanson, «d'ac-

3. «Symbolicité» traduit symbolicity. Il est avéré en français au moins depuis l'ouvrage d'Angèle Kremer-Marietti intitulé précisément La Symbolicité, essai sur les formes de la symbolisation (Paris, Puf, 1982). Je n'en connais que la version résumée par son auteur dans l'article Burke, Perelman, Lacan et la métaphore, lisible sur www.psychanalyse.lu. Mais il me semble qu'il s'agit d'une orientation trop psychanalytique et pas assez politique du dramatisme. 
centuer le positif», et quand certains experts en communication gagnent beaucoup d'argent en écrivant des œuvres à succès qui font l'éloge du "pouvoir du penser positif », la deuxième clause de ma définition doit se charger de la tâche difficile et ingrate de célébrer cette merveille spécifiquement humaine, le négatif. J'ai dit ailleurs quel révélateur avait été pour moi le chapitre «L'idée de néant» dans L'Évolution créatrice de Bergson 4 . Il m'a amené à réaliser qu'il n'y a pas de négatifs dans la nature, où tout est simplement ce qu'il est et tel qu'il est. [...]

Toutefois, je ferais un changement d'orientation par rapport au chapitre fertile de Bergson. Son approche est un peu trop «scientiste» pour des intentions spécifiquement «dramatistes». Ainsi, en mettant l'accent sur des problèmes de connaissance, il accentue le négatif propositionnel, «Ce n'est pas». Dramatistiquement, l'accent devrait être sur le négatif normatif: «Tu ne dois pas» (Thou shalt not) $)^{5}$. Le négatif ne se présente pas comme une source de définition ou d'information, mais comme un ordre, comme «Ne fais pas cela» (Don't). Ses potentialités plus «scientistes» se développent plus tard. Et alors que Bergson a raison d'observer que nous ne pouvons avoir une «idée de néant» (que nous devons imaginer une tache noire, ou quelque chose qui est annihilé, ou un abîme), je suggère que nous pouvons avoir une «idée de Non», une «idée de ne fais pas cela ». [...]

En un sens, il y a un paradoxe dans «ne fais pas cela». Car le négatif est seulement un principe, une idée, et non un mot pour une chose. Et ainsi, alors qu'une injonction comme «tu ne dois pas tuer» peut se comprendre au moins comme une idée négative, elle porte aussi en elle l'image positive du tuer (of killing). Mais le point principal est celuici : quoiqu'un enfant puisse ne pas toujours obéir au «tu ne dois pas», et quoiqu'il puisse inévitablement se trouver, à l'horizon, une image

\footnotetext{
4. L'analyse scientiste de l'idée de néant, dans L'Évolution créatrice, est lisible dans Bergson, CEuvres, Puf, p. 728-747.

5. «Tu ne dois pas» efface la connotation biblique de «Thou shalt not». Mais «Tu ne ... ras point» était frustrant, et j'ai été inspiré par Michel Foucault en personne, qui abrège par cette formule les conceptions exclusivement répressives de la domination dans la conférence intitulée «Les mailles du pouvoir» (Dits et écrits, Paris, NRF, 1995, t. IV, p.182). Celle-ci ne vise d'ailleurs pas le dramatisme, apparemment ignoré de Foucault. Au bilan, Burke ne me semble pas être prisonnier de l'hypothèse répressive, comme le prouve bien sa théorie de la stimulation: dans sa perspective, dominer n'est pas seulement interdire, c'est aussi inciter (et pas seulement à ce qu'on interdit).
} 
qui invite positivement à la désobéissance, l'enfant «comprend la leçon».

Troisième clause: séparé de sa condition naturelle par des instruments de sa propre fabrication. [...]

Cette clause est conçue pour tenir compte de ceux qui définissent l'homme comme «l'animal qui se sert d'outils» (homo faber, homo economicus et autres). Ceux qui mettent initialement l'accent sur les outils définissent ensuite le langage lui-même comme une espèce d'outil. Mais quoique l'instrumentalité soit un important aspect du langage, nous ne pouvons pas la traiter proprement comme l'essence du langage. Le langage est une espèce d'action, action symbolique - et sa nature est telle que l'on peut s'en servir comme d'un outil [...].

Quatrième clause: stimulé par l'esprit de hiérarchie. Ici, l'usage de symboles par l'homme se combine avec sa négativité et ses tendances à une différenciation des moyens de subsistance implicite dans les inventions qui favorisent la division du travail, le résultat étant des définitions, des différenciations et des répartitions de la propriété protégées par les négativités de la loi. [...]

Pendant longtemps, j'ai pensé que ces clauses épuisaient largement le sujet. Pourtant, pour des raisons encore inexpliquées, j'ai décidé qu'un codicille de fin était encore nécessaire, ce qui nous donne, en somme :

\section{L'homme est}

l'animal qui se sert de symboles (qui les fabrique et qui s'en sert mal),

inventeur du négatif (ou civilisé par le négatif),

séparé de sa condition naturelle par des instruments de sa propre fabrication,

stimulé par l'esprit de hiérarchie (ou animé par le sens de l'ordre), et pourri par la perfection.

Je dois vite expliquer et justifier ce codicille désabusé. 
Le principe de perfection est central dans la nature du langage comme motif. Le simple désir de désigner quelque chose par son nom «exact» ou de parler une langue dans ses tours caractéristiques est intrinsèquement "perfectionniste». Et qu'y a-t-il de plus essentiellement «perfectionniste» que cette envie, quand on a grand besoin de quelque chose, de formuler ce besoin de telle sorte que l'on «définit» réellement la situation? Même un poète qui met au point d'habiles procédés de distorsion du langage le fait avec des principes perfectionnistes en tête. [...]

Pour y voir plus clair, il suffit d'élargir le concept de perfection au point où nous pouvons aussi nous servir ironiquement de ce terme, comme quand nous parlons d'un «parfait crétin» ou d'un «parfait coquin ». Et bien sûr, j'avais précisément ces possibilités en tête quand dans mon codicille je fais référence à l'homme comme étant pourri par la perfection.

L'aspect ironique de ce principe se manifeste lui-même à la perfection dans notre tendance à concevoir un «ennemi parfait». La version nazie du Juif, telle qu'elle se présente dans le Mein Kampf de Hitler, est l'exemple le plus achevé de cette «perfection» ironique à une époque récente, quoique des tendances similaires ne cessent d'apparaître dans les controverses en cours entre «l'Est»et «l'Ouest».

\section{Une grammaire des motifs (1945)}

\section{Sur la clarté 6}

Un perfectionniste pourrait chercher à élaborer des termes dénués de toute ambiguïté ou inconséquence (comme dans l'idéal terminologique de la logique symbolique ou du positivisme logique). Mais nous avons un objectif différent en vue [...]. Nous présupposons que dans la mesure où les hommes ne peuvent créer eux-mêmes l'univers, il doit rester quelque chose d'essentiellement énigmatique dans le problème des motifs, et que cette énigme sous-jacente se manifeste dans d'inévitables ambiguïtés et inconséquences parmi les termes des motifs.

6. A Grammar of Motives (GM), [UCP, 1969), Introduction, p. XVIII. 
Ainsi, ce que nous voulons, ce ne sont pas des termes qui évitent l'ambiguïté, mais des termes qui révèlent clairement les points stratégiques où les ambiguïtés surgissent nécessairement.

\section{Sur la création ${ }^{7}$}

La création comme acte de Dieu fut une nouveauté totale; et elle fut magique parce que, de même que le magicien voudrait faire croire qu'il tire un lapin vivant d'un chapeau vide, de même Dieu fit tout à partir de rien. Le magicien voudrait nous faire penser qu'il suspend les lois du mouvement. Et l'acte de Dieu, semblablement, «suspendit» les lois du mouvement, quoique dans un sens absolu: c'est-à-dire que de son acte originaire dépendent toutes les lois du mouvement que les hommes acceptent nécessairement comme les conditions du mouvement. En vérité, l'analogie suggère la pensée que la «vraie» magie prévaut en dehors du royaume strict du mouvement, dans le domaine du plus-quemouvement que nous appelons action. L'exigence d'un genre de magie humaine qui viole la loi naturelle se révèle alors comme un idéal superstitieux et quasi-scientifique. Mais la magie au sens de nouveauté semble exister normalement, à un certain degré, comme ingrédient de tout acte humain; car chaque acte contient une certaine quantité de motivation qui ne peut être expliquée simplement dans les termes du passé, étant dans une certaine mesure, même minuscule, du nouveau (a new thing).

\section{Sur l'argent ${ }^{8}$}

Quand le motif capitaliste devint la norme, les hommes purent « de leur plein gré » entrer en compétition pour des émoluments monétaires; ainsi ils purent «librement» exécuter toutes sortes d'actes nécessaires, dont une grande partie étaient si nouveaux et étrangers aux traditions de la culture occidentale qu'ils auraient semblé totalement irrationnels à l'aune des normes de la seule coutume. Ils étaient également irrationnels à l'aune des exigences de satisfaction intrinsèque au travail, et pouvaient être rationalisés uniquement au point de vue de l'argent gagné,

7. $G M$, p. 65.

8. $G M$, p. $92-93$. 
aussi minime fût-il. Nous eûmes ainsi le spectacle d'hommes libres en concurrence les uns avec les autres pour obtenir un travail qui était intrinsèquement très déplaisant, n'ayant rien d'autre en sa faveur que la rétribution monétaire extrinsèque; les hommes s'engageaient pour des tâches que dans les scènes économiques antérieures ils auraient été amenés à exécuter seulement sous la contrainte, comme des esclaves ou des détenus, ou par des motifs aussi rares que le service volontaire tel qu'on le trouve dans les allégeances personnelles ou familiales.

En somme, si vous devez mener à bien du travail déplaisant et si vous ne voulez pas le faire vous-même, dans une culture de l'esclavage vous pouvez le faire faire de force, sous la contrainte, sous la menace. Dans une culture de la piété vous pouvez le faire faire «religieusement », si ceux à qui vous demandez de faire le travail sont animés par des motifs tels que la dévotion, l'admiration, le sens du devoir. Mais dans un marché du travail capitaliste, il vous suffira de dire: "Qui veut le faire pour cinq dollars? », et les hommes accourront «indépendamment», de leur «plein gré», sous les ordres de personne, pour s'enrôler «volontairement» dans ce travail ${ }^{9}$.

\section{Une rhétorique des motifs (1950)}

\section{Identification et division ${ }^{10}$}

La Rhétorique a affaire aux possibilités de la classification dans ses aspects partisans; elle considère les façons dont les individus sont en conflit, ou s'identifient avec des groupes plus ou moins en conflit.

Pourquoi «en conflit», demandera-t-on, quand le terme titulaire est «identification»? Parce que commencer par l'«identification», c'est, par là même, quoique indirectement, faire face aux implications de la

9. La critique du sens du devoir professionnel à tout prix a également été portée avec une grande vigueur par l'écrivain Günter Anders, notamment dans ses lettres ouvertes au fils d'Eichmann écrites peu après la pendaison du fonctionnaire nazi à Jérusalem. Elles ont été traduites en français et rassemblées sous le titre Nous, fils d'Eichmann (Paris, Rivages, 2003). Anders a également écrit à Claude Eatherly, le «pilote d'Hiroshima» (en fait l'homme qui a transmis le go ahead aux pilotes d'Hiroshima) quand celui-ci était interné dans un «Veterans Hospital».

10. A Rhetoric of Motives (RM), [UCP, 1969), p. 22 et 25-26. 
division. Et ainsi, finalement, les hommes sont amenés à la plus tragiquement ironique de toutes les divisions, dans laquelle des millions d'actes coopératifs vont vers la préparation d'un seul acte destructeur. Nous faisons allusion à cette ultime maladie de la coopération: la guerre. (On comprendra beaucoup mieux la guerre si l'on ne la pense pas simplement comme une dissension portée à son point critique, mais plutôt comme une maladie, une perversion de la communion. La guerre moderne requiert de façon caractéristique une myriade d'actes constructifs pour chaque acte destructeur; avant chaque explosion finale il doit y avoir un vaste réseau d'opérations imbriquées et dirigées en commun.)

L'identification est affirmée avec vigueur précisément parce qu'il y a de la division. L'identification compense la division. Si les hommes n'étaient pas séparés les uns des autres, il n'y aurait pas besoin d'un rhétoricien pour proclamer leur unité. Si les hommes étaient entièrement et vraiment d'une seule substance, la communication absolue appartiendrait à l'essence même de l'homme. Elle ne serait pas un idéal, comme elle l'est maintenant, en partie incarné dans des conditions matérielles, en partie frustré par ces mêmes conditions; elle serait plutôt aussi naturelle, spontanée et totale que chez ces prototypes idéaux de la communication, les anges, ou les «messagers», des théologiens. [...]

Dans la pure identification, il n'y aurait pas de dissension. De même, il n'y aurait pas de dissension dans la séparation absolue, puisque des opposants ne peuvent entrer en lutte qu'à travers un terrain médiateur qui rend leur communication possible, fournissant ainsi la première condition nécessaire à l'échange de coups. Mais si l'on relie l'identification et la division de façon ambiguë, de sorte que l'on ne peut savoir au juste où l'une commence et où l'autre finit précisément, l'on tient alors l'invitation caractéristique à la rhétorique. C'est là l'une des raisons principales pour laquelle la rhétorique, selon Aristote, "prouve les contraires». Quand deux hommes collaborent dans une entreprise à laquelle ils contribuent par différentes sortes de services et dont ils tirent différentes quantités et sortes de profit, qui peut dire, une fois pour toutes, où la «coopération» finit et où l'«exploitation » d'un partenaire par l'autre commence précisément? La ligne indécise entre les deux ne peut être «scientifiquement» identifiée; des rhétoriciens rivaux peuvent la tracer à différents endroits, et leur pouvoir persuasif (persuasiveness) varie selon les ressources 
que chacun a à sa disposition. (Quand des questions publiques sont en jeu, ces ressources ne sont pas limitées aux capacités intrinsèques du locuteur et du discours, mais leur efficacité dépend aussi des moyens purement techniques de communication, qui peuvent soit aider l'expression soit la gêner. Car une «bonne» rhétorique négligée par la presse ne peut évidemment pas être aussi «communicative» qu'une rhétorique pauvre soutenue dans tout le pays par des gros titres. Et nous devons souvent penser la rhétorique non pas en termes d'allocution particulière, mais comme un vaste corps d'identifications qui doivent bien plus leur force de conviction [convincingness] à la répétition triviale et à la morne insistance quotidienne qu'à une habileté rhétorique exceptionnelle.)

\section{Sur la spécialisation ${ }^{11}$}

Le fait qu'une activité soit capable d'être réduite à des principes intrinsèques, autonomes ne prouve pas qu'elle est libre d'être identifiée avec d'autres ordres de motivation qui lui sont extrinsèques. Ces autres ordres lui sont extrinsèques si on les considère du point de vue de l'activité spécialisée seule. Mais ils ne sont pas extrinsèques au champ de l'action morale comme telle, considérée du point de vue de l'activité humaine en général. L'agent humain en tant qu'agent humain n'est pas motivé seulement par les principes de l'activité spécialisée.

L'identification inavouée par laquelle une fonction théologique est introduite dans un terme en apparence entièrement laïque peut secrètement renforcer le principe typiquement libéral de l'autonomie professionnelle, lui-même renforcé par l'idée naïvement pragmatiste que le travail pratique spécialisé est un fondement suffisant de la moralité. Si l'on assigne à l'expert technicien, en tant que tel, la tâche de perfectionner des nouvelles puissances de destruction chimique, bactériologique ou atomique, sa moralité en tant qu' expert requiert seulement qu'il s'applique à sa tâche aussi efficacement que possible. Si l'on demande ce que la nouvelle force pourrait signifier, une fois diffusée dans une structure sociale émotionnellement et intellectuellement inapte à la contrôler, ou une fois livrée à des hommes dont la spécia-

11. $R M$, p. $30-31$. 
lité est le meurtre professionnel, eh bien, cela n'est simplement "pas son affaire» en tant que spécialiste, quelles que soient ses appréhensions en tant que père de famille, ou en tant que citoyen de sa nation ou du monde. L'extrême division du travail sous le libéralisme capitaliste récent ayant fait de la dispersion la norme et ayant transformé l'état de Babel en idéal, le vrai libéral doit voir presque comme un affront le souci rhétorique des identifications par lesquelles les principes d'une spécialité ne peuvent pas être jugés sur leur apparence, simplement comme les motifs propres à cette spécialité. Ils sont les motifs propres à la spécialité en tant que telle, mais pas à la spécialité en tant que participant à un contexte de motifs plus large.

\section{Sur l'idéologie ${ }^{12}$}

Ces mêmes journaux qui sont publiés pour de l'argent, qui reçoivent leur revenu en faisant de la publicité pour des biens vendus pour de l'argent, qui sont lus par les gens en direction ou au retour du lieu où ils travaillent pour de l'argent et qui distribuent des comptes rendus d'événements politiques, économiques et sociaux qui ont à voir avec de l'argent (notamment des nouvelles au sujet des crimes contre la propriété) - ces mêmes journaux, dans leurs moments les plus édifiants, parleront plutôt de la «liberté», de la «dignité de l'individu», de «l'homme occidental», de la «civilisation chrétienne», de la «démocratie» et ainsi de suite, comme les motifs obligeant au moins notre peuple et notre gouvernement, et dans une moindre mesure les «nations » que «nous » voulons comme alliés, mais pas la classe dominante, ou la clique qui domine les pays avec lesquels «nous» sommes en conflit.

\section{Sur le mysticisme ${ }^{13}$}

Le mysticisme n'est pas denrée rare. Certes, on ne le trouve que rarement à l'état pur. Et ses analogues laïcs, dans un symbolisme grandiose ou gracieux, sont rares. Mais on en éprouve le besoin, la démangeaison, en tout lieu. Et ce besoin s'intensifie avec la hiérarchie. [...] 
Puisque, pour le meilleur et pour le pire, le mystère du hiérarchique est en permanence devant nous, examinons donc minutieusement, comme des étudiants en rhétorique, sa capacité à nous mettre en transe, à la fois avec consternation et délice. Et remarquons finalement, tout autour de nous, nous stimulant en permanence, quoique par fragments, le motif qui trouve son identification ultime dans la pensée, non pas de l'holocauste universel, mais de l'ordre universel - comme dans la symétrie rhétorique et dialectique de la métaphysique aristotélicienne, où toutes les classes d'êtres s'arrangent hiérarchiquement dans une chaîne, une échelle, ou une pyramide de valeur croissante, chaque genre s'efforçant d'accéder à la perfection de son genre, et ainsi d'accéder au genre juste au-dessus de lui, jusqu'à ce que (while) les efforts de la série entière culminent en Dieu, la cynosure et sinécure bien-aimée qui achève tout désirit ${ }^{14}$.

14. «Cynosure» reproduit cynosure, pour maintenir l'euphonie avec «sinécure», qui reproduit plus légitimement sinecure. Kunosoura a pu signifier «Petite Ourse» en grec, et sa version anglaise « Étoile polaire», «point de mire» ou «centre d'attraction».

*

Je remercie Thibault Lemonde d'avoir le premier devant moi introduit Kenneth Burke dans une conversation. Lui-même remercie son professeur Henri Meschonnic de l'avoir introduit à Kenneth Burke dans Politique du rythme, politique du sujet (Lagrasse, Verdier, 1995, p. 385-406). Enfin, je remercie Renaud Pasquier et Laurence Marie pour leur hospitalité et leur sens critique. 